form different sources into a pathological tissue response. Resorption of the juxtaarticular bone is part of this pathological tissue response and is denoted as focal bone erosion which occurs early in this disease and over time is associated with significant morbidity. Focal bone erosions are observed at the interface of pannus and bone tissue both marginally, where pannus invades cortical bone, and in the immediate subchondral bone, where the pannus invades the marrow space. Many of the cytokines and growth factors implicated in the inflammatory processes are secreted by FLS and have also been demonstrated to impact directly or indirectly on osteoblast and/or osteoclast differentiation and function. However, research of the last years has also identified some novel pathways by which i) osteoclast- mediated bone resorption is regulated and fine-tuned under inflammatory conditions such as in RA and that ii) link inflammatory bone resorption to other features of systemic autoimmunity such as the activation of developmental pathways or muscle weakness. This lecture will review some of these novel mediators and pathways, including members of the Wnt- signalling pathway (e.g. sclerostin), members of the GDF- family of growth differentiation factors (e.g. myostatin) and cells surface anchored proteoglycans (e.g., syndecans). Focusing on the role of these molecules in the FLS-mediated regulation of osteoclastic bone resorption, the lecture will point to general principles of inflammatory bone remodelling and discuss potential therapeutic implications for RA.

Disclosure of Interest: None declared

DOI: 10.1136/annrheumdis-2017-eular.7261

\section{SP0136 MULTIFACTORIAL TISSUE GROWTH FACTORS}

\section{C.P. Denton. Centre for Rheumatology, Royal Free Hospital and Ucl Medical School, London, United Kingdom}

Connective tissue is a critical component of all organs and has special importance in the musculoskeletal system. In specialist tissues, such as bone, cartilage of muscle an in vascular structures and internal organs there is a mixture of less specialized connective tissue together with the specific tissue and organ specific cellular components. In addition to physiological function there are two other areas in which it plays a central role. Normal embryonic an postnatal growth and development, and tissue repair in response to injury. It has emerged that there are shared pathways, mediators and mechanisms in these different processes and that multifactorial tissue growth factors have an important role. In this way, these proteins can have a profound influence on multiple cell types and provide a mechanistic link in many complex chronic diseases. It is notable that pathways that are perturbed in one chronic disease, such as atherosclerosis or osteoarthritis may be similarly disrupted in different context in conditions such as lung fibrosis or scleroderma. Transforming growth factor beta family members are prototypic examples of this class of protein. This included TGFbeta isoforms but also the related bone morphogenetic (BMP) and activin family of proteins. They share chemical structure and an ability to regulate multiple cell types in a context specific way. In addition, there is remarkable complexity in their regulation with intrinsic inhibitory mechanisms to protect from inappropriate biological activity. Their role in repair means that activation of preformed protein may regulate biological effects. Proteins regulated by the TGFbeta family, such as the CCN family of matricellular proteins and some for the cardinal growth factors and cytokines share properties. There is functional and signaling redundancy and cross talk. Conceptually it is interesting to envisage dysfunctional networks of cytokines in disease that may be attenuated by extracellular blocking or antagonism. Insights for many areas of developmental biology and pathology have informed about this challenging area of molecular medicine.

Disclosure of Interest: C. Denton Grant/research support from: Inventiva, CSL Behring, GSK, Bayer, Consultant for: GSK, Actelion, Inventiva, Roche DOI: 10.1136/annrheumdis-2017-eular.2532

\section{SP0137 FROM GLYCOSYLATION TO INFLAMMATION}

\section{Herrmann. University Hospital Erlangen, Erlangen, Germany}

One of the best studied posttranslational modification of immunoglobulin G ( $\lg G)$ is the addition of a complex glycan to asparagine 297 , which is required for IgG to bind to Fc-receptors and for the activation of complement via the classical activation pathway. In the absence of the glycan the $\lg G$ usually behave like a lame duck unable to execute its effector functions. The sugar tree is polymorphic and there are several functional consequences related to the glycan. The terminal sialylation, the presence of galactose, the core fucosylalation and the insertion of a bisecting third sugar chain all reportedly modify the biological and functional activities of IgG.

As examples for the impact of Asp-297 associated glycans to the activity I will present (I) the sialylation of anti-histon autoantibodies in patients with systemic lupus erythematosus (II) the exposure of sugar epitopes by random IgG isolated from patients with rheumatoid arthritis (III) the molecular analysis of the glycan of random IgG isolated from patients with inflammatory diseases and controls and (IV) and finally the glycosylation of IgG-anti-phospholipid autoantibodies in healthy children.

(I) The autoantibodies are mainly found in the non-sialyated fraction of the anti-histon autoantibodies which can be considered inflammatory lgG.

(II) The circulating random IgG from patients with rheumatoid arthritis show an increased exposure of the Asp-297 associated glycans.

(III) The molecular analysis of the glycan of random IgG isolated from patients with systemic lupus erythematosus, rheumatoid arthritis, sepsis showed diseasespecific glycans.

(IV) The non-pathogenic IgG-anti-phospholipid autoantibodies isolated from healthy children displayed an increased sialylation when compared to the pathogenic autoantibodies from adults with anti-phospholipid syndrome.

The examples point to a strong impact of IgG glycosylation in the etiopathogenesis of chronic inflammatory rheumatic diseases. One should consider to include IgG glycan analyses into the diagnostic repertoire for autoantibodies.

Disclosure of Interest: None declared

DOI: 10.1136/annrheumdis-2017-eular.7291

\section{FRIDAY, 16 JUNE 2017}

\section{Patient engagement in research: best practices,} benefits, and challenges

\section{SP0138 THE CHALLENGES OF PATIENT INVOLVEMENT IN SCIENTIFIC RESEARCH. HOW TO ACHIEVE TRUE REPRESENTATION OF THE PATIENTS' VOICE?}

M. De Wit on behalf of the EULAR Working group for Collaborative Research. Dept. Medical Humanities, EMGO+ research institute, VU University Medical Centre, Amsterdam, Netherlands

There is a strong call in the western world for collaborative research. Rheumatology is one of the disciplines that has explored best practices and paved the way to implement lessons learned. Although good methodologies for evaluating the impact of patient participation stay behind, we have been able to develop expertbased recommendations for collaboration. They are helpful for both researchers and patient representatives, but they do not address all concerns that occur in daily practice. In this lecture current challenges of integrating the perspectives of patients in clinical research will be discussed: 1. Defining the role and complementary value of patient research partners; How can patients in the role of collaborative partner contribute to research in addition to the data that patients provide as respondent or study participant? 2. Facilitating and guiding researchers in collaborative research; We have experience in preparing and educating patient representatives for their role in research, but how should researchers be trained and supported? 3. Preserving the patients' voice throughout the research process; Qualitative research may elicit patients' experiences with health care or health interventions, but what is needed to guarantee that these findings are not lost in the rigorous process of producing scientific knowledge? 4. Demonstrating impact of collaborative research to funders and the public; Despite many case studies reporting a great variety of patient participation methods, there are no clinical trials nor consensus on a validated methodology for assessing the outcomes of patient involvement in research.

For each challenge we will try to propose a way forward. These challenges will also be discussed at the newly established EULAR working group for collaborative research.

Disclosure of Interest: None declared

DOI: 10.1136/annrheumdis-2017-eular.7249

\section{SP0139 MEANINGFUL PATIENT AND PUBLIC INVOLVEMENT IN PLANNING RESEARCH}

A. Redmond. Leeds Institute for Rheumatic and Musculoskeletal medicine, University of Leeds, Leeds, United Kingdom

Historically, services providing care for people with long term conditions (LTCs), together with any associated research agendas, have been driven entirely by clinicians. In many cases this is well meaning and is done with some consideration of what patients need, but almost always the end result relies on the clinicians' interpretation of what they think patients need.

Recently it has become more usual to involve patents in planning research projects and service changes directly. Initial attempts to give patients genuine input were ad hoc in nature and so patient contributions could be lacking, ignored or misinterpreted.

In many cases having poor patient input is worse than no input at all as it lends false credibility to the process. Lately a range of formal requirements, arrangements and methods have become adopted and this presentation will focus on how these can be brought to bear to ensure meaningful patient and public involvement in planning research. From national guidance such as the UK's INVOLVE initiative, to local standard operating procedures we will share experiences and lessons learned.

The session will close with a practical summary of how to make PPI contributions genuine and meaningful, and some insights into possible future developments as this aspect of the research process matures and consolidates.

Disclosure of Interest: None declared

DOI: 10.1136/annrheumdis-2017-eular.7220 